\title{
Risk Factors of Biochemical Failure in Locally Advanced Carcinoma Prostate Treated With Definitive External Beam Radiotherapy and Androgen Deprivation Therapy: Experience From Tertiary Care Center in North India
}

\author{
Manjinder Sidhu ${ }^{1}$, Davinder Paul ${ }^{2}$, Sandhya Sood ${ }^{1}$, Kunal Jain ${ }^{2}$, Jagdeep Singh ${ }^{2}$, Ritu Aggarwal ${ }^{1}$, \\ Divyaanshi Sood ${ }^{3}$ \\ 1. Radiation Oncology, Dayanand Medical College \& Hospital (DMCH) Cancer Center, Ludhiana, IND 2. Medical \\ Oncology, Dayanand Medical College \& Hospital (DMCH) Cancer Center, Ludhiana, IND 3. Oncology, Dayanand \\ Medical College \& Hospital (DMCH), Ludhiana, IND
}

Corresponding author: Sandhya Sood,drsandhyasood@gmail.com

\section{Abstract}

\section{Background}

Locally advanced prostate cancer (LACAP), despite external beam radiotherapy (EBRT) along with antiandrogen therapy (ADT) has risk of prostate-specific antigen (PSA) progression. Furthermore, number of studies have emphasized on different prognostic factors. The purpose of our study is to analyze risk factors for biochemical failure (BF) in these patients treated at our institute.

\section{Methods}

Our study is a single-institution retrospective observational done at a tertiary care center in North India. Between January 2018 and December 2020, we retrospectively identified 34 patients managed at our institute as per multidisciplinary board (MBD). Demographic, clinical, radiological, pathological and treatment-related parameters were assessed as potential risk factors. End-point of the study was to find significant risk factors for BF. Statistical analysis was done on SPSS, version 20 (IBM Corp., Armonk, NY).

\section{Results}

All eligible patients received EBRT with ADT as per institution policy. Mean follow-up period was 20 months during which two (5.9\%) patients had BF at a mean of 30 months after EBRT. Four-year PSA-progressionfree survival rate was $73 \%$. On univariate analysis, prognostic factors associated with high risk of BF were Gleason score and clinical T stage.

Review began 07/24/2021 Review ended 07/29/2021 Published 08/04/2021

\section{(c) Copyright 2021}

Sidhu et al. This is an open access article distributed under the terms of the Creative Commons Attribution License CC-BY 4.0., which permits unrestricted use, distribution, and reproduction in any medium, provided the original author and source are credited.

\section{Conclusion}

In summary, prognostic factors for high risk of BF leading to clinical progression are Gleason score 9 or 10 and clinical T3b stage.

\section{Categories: Oncology}

Keywords: locally advanced prostate cancer, external beam radiotherapy, antiandrogen therapy, prognostic factors, biological failure

\section{Introduction}

In India annual prostate cancer incidence rate ranges from 5.0 to 9.1 per 100,000/year [1], but also of all, $85 \%$ are detected late (stages III and IV). Furthermore, recommended treatment for locally advanced prostate cancer is external beam radiotherapy (EBRT) and antiandrogen therapy (ADT) as supported by the literature [2-4]. Nevertheless, a significant minority will eventually relapse [5,6]. It is important to note that the rise of prostate-specific antigen (PSA) above a defined threshold, which is called biochemical failure (BF), is usually the earliest harbinger of replaced disease after radiotherapy, and may manifest years prior to clinical recurrence [7].

Some of the high-risk factors in carcinoma prostate which have already been studied in various reports are: extent of the primary tumor (clinical stage), nodal status, degree of histological differentiation, zone of origin and serum PSA levels [8-11].

In this article, we retrospectively analyzed prognostic factors precisely leading to $\mathrm{BF}$ in our group of locally advanced prostate cancer (LACAP) prostate treated with standard EBRT and ADT. 


\section{Materials And Methods}

\section{Study design}

Single institution cross-sectional retrospective analysis. The requirement for ethical approval was waived due to the retrospective study design.

\section{Study place}

The study was done at a tertiary care center in North India

\section{Study period}

Medical records of patients were collected from electronic medical record (EMR) available in Aria platform (version 16.1, CTSH [Cancer Treatment Services Hyderabad Pvt.], Ludhiana, Punjab) from January 2018 to December 2020. The last follow-up was May 2021.

\section{Study population}

Thirty-four biopsy-proven adenocarcinoma prostate referred to our department which met the criteria were included in the study. Inclusion criteria were men with clinical stage T3 to T4 adenocarcinoma prostate treated with definitive radiotherapy and ADT. Patients with positive lymph nodes were eligible if the involved nodes remained below the common iliac level. Early and Metastatic disease was excluded from the study.

\section{Pre-treatment evaluation}

All patients underwent physical examination, Karnofsky performance status evaluation, routine laboratory studies including serum prostate-specific antigen (PSA) and radiological workup included chest X-ray, bone scan or Positron Emission Tomography and Computed Tomography (PET-CT) scan (model: Discovery IQ, Make: GE Healthcare, Ludhiana, Punjab)

\section{Procedure}

Radiation Simulation, Planning and Delivery

All patients underwent immobilization in supine position on all-in-one immobilization system with both arms kept aside. Moreover, as per institution policy bladder filling protocol was followed, as well as it was ensured that rectum was empty during simulation. Contrast Computed tomography (CT) images (Discovery IQ, Make: GE Healthcare) were taken of lower abdomen and pelvis at 2.5-mm slice thickness. CT images were imported and contoured in Eclipse planning system version 16.1 (Varian Medical System, Palo Alto, CA).

For target delineation, we followed the Radiation Therapy Oncology Group (RTOG) contouring atlas for prostate cancer [12]. In addition, guidelines were also followed for pelvic nodal delineation [13]. Organ at risk delineated were: rectum, bladder, bowel and femoral heads. Entire rectum from the anal verge up to the recto-sigmoid junction was contoured [14-16]. Besides whole organ delineation, bladder wall and rectal wall were contoured using a 5-mm internal margin. Constraints for organs at risk were as follows: rectal wall: volume receiving $60 \mathrm{~Gy} \leqslant 50 \%$ and volume receiving $70 \mathrm{~Gy} \leqslant 20 \%$; bladder wall: volume receiving $65 \mathrm{~Gy} \leqslant 50 \%$; and femoral heads: volume (left, right) each receiving $50 \mathrm{~Gy} \leqslant 10 \%$. Treatment plans were generated using the TPS. All patients underwent rapid-arc planning. Patient underwent treatment on True Beam Linear accelerator (Varian Medical System, Palo Alto, CA) coupled with daily Cone-beam computed tomography systems (CBCT) for all patients. Daily CBCT of all patients were taken. Prescribed dose to PTV was 70 Gy in 35 fractions. Furthermore, pelvic lymph node received 50.4Gy in 28 fractions by SIB technique. Photon optimizer, version 13.7.16 was used for inverse optimization with $2.5 \mathrm{~mm}$ optimization resolution. For calculation, anisotropic analytical algorithm (version 13.7.16) was used and the calculation grid was $2.5 \mathrm{~mm}$. Jaw-tracking option was selected to reduce the MLC leakage dose and inhomogeneity correction was applied for all plans. The isocenter was placed at center of PTV volume. The dose was prescribed such that $>95 \%$ of the planning target volume received $100 \%$ of the prescribed dose. The rectal volume receiving $>65 \mathrm{~Gy}$ and $>50$ Gy was limited to $<17 \%$ and $<35 \%$, respectively. Likewise, bladder volume receiving $>65$ Gy and $>40$ Gy was limited to $<25 \%$ and $<50 \%$, respectively. Furthermore, small bowel (peritoneal cavity) constraints were V45Gy $<195 \mathrm{cc}[17]$.

Androgen Deprivation Therapy

Androgen deprivation was used at the discretion of the physician. All patients received ADT before, during and after the planned course of radiotherapy. ADT consisted primarily of an oral antiandrogen and luteinizing hormone-releasing hormone agonist administered as subcutaneously depot injections.

Follow-up 


\section{Cureus}

Generally, follow-up examinations were performed initially at every three months after radiotherapy treatment during the first year, and subsequently at six-month intervals with serial PSA determination and physician-performed digital rectal examination at each visit. BF was defined as a rise of $2 \mathrm{ng} / \mathrm{mL}$ or above the nadir PSA after EBRT with or without hormonal therapy [18]. The interval to BF (IBF) was defined as the time from completion of radiotherapy to BF. Distant metastasis (DM) was defined as metastasis in the bones, visceral organs, or lymph nodes outside of the pelvis. Imaging coupled with biopsy was done as per the discretion of the treating physician.

\section{Study outcomes}

The endpoint was to evaluate significant prognostic risk factors for BF. PSA-progression-free survival rate was defined as the time from date of end of treatment to date of event defined as first documented BF as per phoenix definition. For the purposes of the current analysis, regional metastasis is defined as clinical or radiographic evidence of involvement of the pelvic lymphatics by the tumour beyond completion of adjuvant $\mathrm{ADT}$. Distant metastases are defined as clinical or radiographic evidence of disease beyond the pelvis during follow-up.

\section{Statistical analysis}

All data were analysed using SPSS Statistics for Windows, Version 20.0 (IBM Corp., Armonk, NY). Generalized linear modelling was done for univariate analysis to establish the association of prognostic factors for the locoregional control. Continuous variables were dichotomized according to their mean values or split into subgroups depending on their clinical significance. Pearson's chi-square test was done for categorical variables. Multivariate analysis by logistic regression was not done in view of insufficient sample size. Survival curves were estimated using the K-M method. For all practical purposes, a p-value of 0.05 or less was considered significant.

\section{Results}

All 34 patients were included for analysis. Baseline clinical, pathologic, and treatment characteristics are detailed in Table 1.

\section{Characteristic}

Mean age(years)

Mean baseline PSA (ng/mL)

Gleason group grade

2

3

4

5

Gleason score

$<=6$

7

8

9 or 10

Gleason pattern

$\leq 3+3$

$3+4$

$4+3$

$4+4,3+5,5+3$

$4+5,5+4,5+5$

Clinical T stage
Number of patients (\%)

68

35.3

$6(17.6 \%)$

$7(20.6 \%)$

$11(32.4 \%)$

$6(17.6 \%)$

$4(11.8 \%)$

$7(20.6 \%)$

$16(47.1 \%)$

$6(17.6 \%)$

$5(14.7 \%)$

$7(20.6 \%)$

$7(20.6 \%)$

9(26.5\%)

6(17.6\%)

$5(14.7 \%)$ 


\section{Cureus}

\section{Clinical N stage}

No

Clinical stage

IIIA

IIIB

IIIC

\section{TABLE 1: Characteristics of the study population.}

Overall, the median age of diagnosis was 68 years (range: 50-76 years) with mean PSA at time of diagnosis $35.3 \mathrm{ng} / \mathrm{mL}$. Most common Gleason group grade was 3. Similarly, Gleason score 7 was commonly seen. Likewise, among Gleason patterns, $4+3$ was slightly more prevalent compared to others. In addition, clinical T3b and N0 represented $50 \%$ and $61.8 \%$ cases respectively. Furthermore, among these locally advanced cases stage IIIB and IVA constituting 76.4\% ( $\mathrm{N}=26)$ of cases. Mean size of prostate tumor was $4 \mathrm{~cm}$ (range 1-6.8cm) with peripheral zone being the most common site in $67.3 \%(\mathrm{~N}=23)$.

All patients received neoadjuvant and adjuvant ADT with a mean of five and 25 months prior to start and completion of radiotherapy respectively coupled with concurrent ADT in all cases. Commonly used hormonal injection was Lupride in $38.2 \%(\mathrm{~N}=13)$ patients. Radiotherapy was delivered by rapid arc technology in all with mean of 70Gy in 35 fractions.

\section{BF and univariate analysis}

Mean follow-up from the end of radiotherapy was 20 months. Two (5.9\%) patients had BF according to Phoenix definition at mean IBF of 30 months after radiotherapy during the study follow-up. For the subset of patients with $\mathrm{BF}$, the median time from $\mathrm{BF}$ to clinically detected metastasis was eight months and out of these one had visceral and other had local recurrence which were confirmed by PET-CT and biopsy respectively. Variable explored for relationship with BF are detailed in Table 2. 


\section{Cureus}

\begin{tabular}{|c|c|c|c|c|}
\hline Variables & Total & BF & No BF & Sig. \\
\hline \multicolumn{5}{|l|}{ Age } \\
\hline$<=68$ & 18(52.9\%) & $1(50 \%)$ & 17(53.1\%) & 0.727 \\
\hline$>68$ & $16(47.1 \%)$ & $1(50 \%)$ & $15(46.9 \%)$ & \\
\hline \multicolumn{5}{|c|}{ Baseline PSA } \\
\hline$<=20$ & 14(53.8\%) & $1(50 \%)$ & $13(54.2 \%)$ & 0.720 \\
\hline$>20$ & 12(46.2\%) & $1(50 \%)$ & $11(45.8 \%)$ & \\
\hline \multicolumn{5}{|c|}{ Gleason score } \\
\hline$<=6$ & $7(20.6 \%)$ & $0(0 \%)$ & $7(21.9 \%)$ & \\
\hline 7 & $16(47.1 \%)$ & $0(0 \%)$ & $16(50 \%)$ & \\
\hline 8 & $6(17.6 \%)$ & $0(0 \%)$ & 6(18.8\%) & \\
\hline $9-10$ & $5(14.7 \%)$ & $2(100 \%)$ & $3(9.4 \%)$ & 0.006 \\
\hline \multicolumn{5}{|l|}{ PNI } \\
\hline Yes & $11(32.4 \%)$ & $0(0 \%)$ & $11(34.4 \%)$ & \\
\hline No & $23(67.6 \%)$ & $2(100 \%)$ & $21(65.6 \%)$ & 0.451 \\
\hline \multicolumn{5}{|c|}{ Size of tumor } \\
\hline$<=4 \mathrm{~cm}$ & $22(64.7 \%)$ & $2(100 \%)$ & $20(65.5 \%)$ & \\
\hline$>4 \mathrm{~cm}$ & 12(35.3\%) & $0(0 \%)$ & 12(37.5\%) & 0.421 \\
\hline \multicolumn{5}{|l|}{ cT stage } \\
\hline T1 & $2(5.9 \%)$ & $0(0 \%)$ & $2(6.2 \%)$ & \\
\hline T2a & $2(5.9 \%)$ & $0(0 \%)$ & $2(6.2 \%)$ & \\
\hline T2c & $1(2.9 \%)$ & $1(50 \%)$ & $0(0 \%)$ & \\
\hline T3a & $3(8.8 \%)$ & $0(0 \%)$ & $3(9.4 \%)$ & 0.004 \\
\hline T3b & $17(50 \%)$ & $1(50 \%)$ & $16(50 \%)$ & \\
\hline T4 & $9(26.5 \%)$ & $0(0 \%)$ & $9(28.1 \%)$ & \\
\hline \multicolumn{5}{|l|}{ c N stage } \\
\hline No & $21(61.8 \%)$ & $1(50 \%)$ & $20(65.5 \%)$ & \\
\hline N1 & 13(38.2\%) & $1(50 \%)$ & $12(37.5 \%)$ & 0.626 \\
\hline
\end{tabular}

TABLE 2: Variables explored for relationship with biological failure.

BF: biochemical failure; PSA: prostate-specific antigen; PNI: perineural invasion.

On univariate analysis by GLIM, the following prognostic factors were associated with high risk of BF, Gleason score X2 (3) $=12.32$, $\mathrm{p}=0.006$ and clinical $\mathrm{T}$ staging $\mathrm{X} 2(5)=17.0, \mathrm{p}=0.004$. Overall, four-year PSAprogression-free survival rate of the cohort was $73 \%$ (Figure 1). 


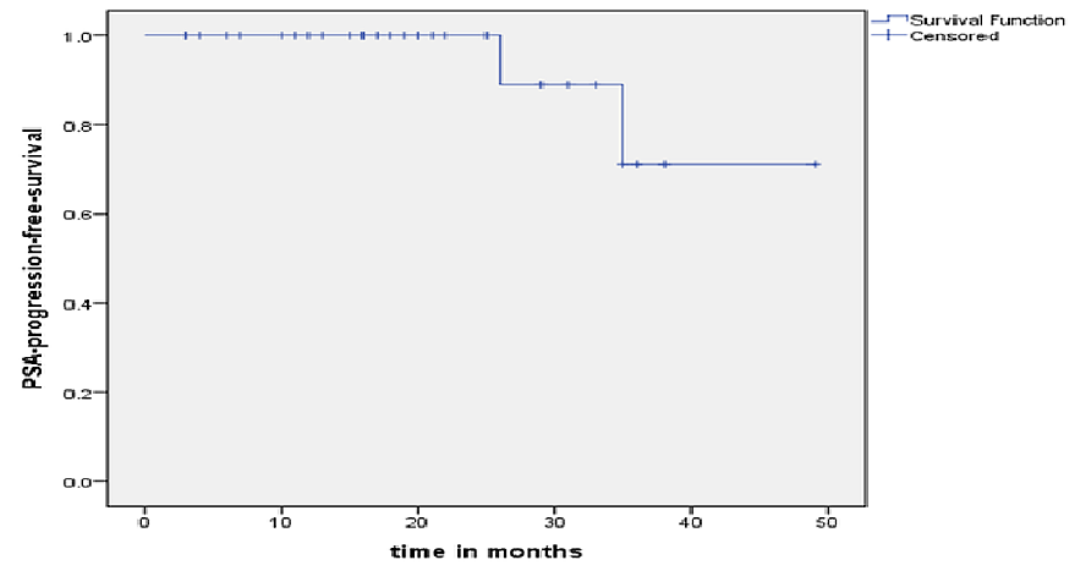

FIGURE 1: Prostate-specific antigen progression-free survival rate.

\section{Discussion}

Our results showed that unfavourable risk factors for early BF in locally advanced carcinoma prostate treated with definitive radiotherapy with ADT are pre-treatment GS 9 or 10 and higher T stage. In these subsets of patient, cases with T3b disease progressed earlier to metastatic disease.

In our study, more than $70 \%$ of patients were of high-risk disease, mainly due to the high initial PSA and Gleason grades at the time of diagnosis. Such finding in our patient characteristics is similar to other reports stating that Asians have a higher tendency for having high-risk disease when matched with the Western population $[19,20]$. Furthermore, these patients are treated at our hospital with RT compared to radical prostatectomy, based on rationale that the outcome is similar when patients are matched by stage and tumour grade [21]. Four-year biochemical free survival rate in our study is $73 \%$. Similarly, for comparison, a compiled report of 34 Japanese institutions reported a 5-year BFS of 71.9\% [22]. It is important to note that patient characteristic and median radiation dose was similar in our study and above Japanese study. In addition, more than $80 \%$ of this series patients were treated with rotation technique similar to our study in which we treated all patients with rapid arc rotational technique. Also, note that all our patients received long-term ADT with mean of 25 months duration which is now standard recommendation for locally advanced carcinoma prostate [23]. Likewise, all our patients received pelvic nodal irradiation despite presently there is a great controversy regarding the effectiveness of elective pelvic radiotherapy in patients with high-risk prostate cancer. On the other hand, the analysis of a recent randomized trial has demonstrated that pelvic irradiation is associated with an improvement in the progression-free survival when neoadjuvant HT is used in conjunction with EBRT [24]. Significant prognostic factors for biochemical recurrence in our study were higher GS and advanced T stage which is supported by various other studies $[21,25]$.

Taken together our findings and findings of previous studies [26] point towards a higher risk of biochemical failure and subsequent clinical progression after EBRT and ADT in locally advanced carcinoma prostate specifically those with GS 9 or 10 and or advanced T stage.

Our study has two major limitations. Firstly, we were not able to do multivariate logistic regression for accurate analysis of predictive factors in view of less sample size. Nevertheless, we were able to address prognostic factors by univariate analysis. Secondly, our analysis was retrospective in nature. Though all data elements were prospectively collected and follow-up was done periodically, there were a lot of lost cases excluded from the analysis. Consequently, the remaining sample eligible for analysis may result in overestimation or underestimation of survival.

\section{Conclusions}

Among various demographic, clinical, radiological, pathological and treatment-related parameters explored for relationship with biological failure in our subset of locally advanced carcinoma prostate patients which were treated by external beam radiotherapy and antiandrogen therapy, the most significant were: pretreatment GS 9 or 10 and higher clinical T stage, precisely T3b. Our results provide potentially clinical useful predictive tools for physicians and patients for locally advanced prostate cancer that necessitates additional measures in these subsets of cases. 


\section{Additional Information \\ Disclosures}

Human subjects: Consent was obtained or waived by all participants in this study. Animal subjects: All authors have confirmed that this study did not involve animal subjects or tissue. Conflicts of interest: In compliance with the ICMJE uniform disclosure form, all authors declare the following: Payment/services info: All authors have declared that no financial support was received from any organization for the submitted work. Financial relationships: All authors have declared that they have no financial relationships at present or within the previous three years with any organizations that might have an interest in the submitted work. Other relationships: All authors have declared that there are no other relationships or activities that could appear to have influenced the submitted work.

\section{References}

1. Hebert JR, Ghumare SS, Gupta PC: Stage at diagnosis and relative differences in breast and prostate cancer incidence in India: comparison with the United States. Asian Pac J Cancer Prev. 2006, 7:547-55.

2. Mason MD, Parulekar WR, Sydes MR, et al.: Final report of the intergroup randomized study of combined androgen-deprivation therapy plus radiotherapy versus androgen-deprivation therapy alone in locally advanced prostate cancer. J Clin Oncol. 2015, 33:2143-50. 10.1200/JCO.2014.57.7510

3. Mottet N, Peneau M, Mazeron JJ, Molinie V, Richaud P: Addition of radiotherapy to long-term androgen deprivation in locally advanced prostate cancer: an open randomised phase 3 trial. Eur Urol. 2012, 62:213-9. 10.1016/j.eururo.2012.03.053

4. Warde P, Mason M, Ding K, et al.: Combined androgen deprivation therapy and radiation therapy for locally advanced prostate cancer: a randomised, phase 3 trial. Lancet. 2011, 378:2104-11. 10.1016/S01406736(11)61095-7

5. Spratt DE, Pei X, Yamada J, Kollmeier MA, Cox B, Zelefsky MJ: Long-term survival and toxicity in patients treated with high-dose intensity modulated radiation therapy for localized prostate cancer. Int J Radiat Oncol Biol Phys. 2013, 85:686-92. 10.1016/j.ijrobp.2012.05.023

6. Rosenbaum E, Partin A, Eisenberger MA: Biochemical relapse after primary treatment for prostate cancer: studies on natural history and therapeutic considerations. J Natl Compr Canc Netw. 2004, 2:249-56. 10.6004/jnccn.2004.0022

7. Lee WR, Hanks GE, Hanlon A: Increasing prostate-specific antigen profile following definitive radiation therapy for localized prostate cancer: clinical observations. J Clin Oncol. 1997, 15:230-8. 10.1200/JCO.1997.15.1.230

8. Pilepich MV, Krall JM, Sause WT, et al.: Prognostic factors in carcinoma of the prostate--analysis of RTOG study 75-06. Int J Radiat Oncol Biol Phys. 1987, 13:339-9. 10.1016/0360-3016(87)90007-1

9. Donohue RE, Fauver HE, Whitesel JA, Augspurger RR, Pfister RR: Prostatic carcinoma influence of tumor grade on results of pelvic lymphadenectomy. Urology. 1981, 17:435-40. 10.1016/0090-4295(81)90183-7

10. Augustin H, Hammerer PG, Blonski J, et al.: Zonal location of prostate cancer: significance for disease-free survival after radical prostatectomy?. Urology. 2003, 62:79-85. 10.1016/s0090-4295(03)00248-6

11. Buhmeida A, Pyrhönen S, Laato M, Collan Y: Prognostic factors in prostate cancer . Diagn Pathol. 2006, 1:4. 10.1186/1746-1596-1-4

12. eContour Team. Intact Prostate Contouring Guide. (2019). Accessed: January 4, 2021: https://econtour.org/training/intact_prostate_module.pdf.

13. Harris VA, Staffurth J, Naismith O, et al.: Consensus guidelines and contouring atlas for pelvic node delineation in prostate and pelvic node intensity modulated radiation therapy. Int J Radiat Oncol Biol Phys. 2015, 92:874-83. 10.1016/j.ijrobp.2015.03.021

14. Fiorino C, Sanguineti G, Cozzarini C, et al.: Rectal dose-volume constraints in high-dose radiotherapy of localized prostate cancer. Int J Radiat Oncol Biol Phys. 2003, 57:953-62. 10.1016/s0360-3016(03)00665-5

15. Greco C, Mazzetta C, Cattani F, Tosi G, Castiglioni S, Fodor A, Orecchia R: Finding dose-volume constraints to reduce late rectal toxicity following 3D-conformal radiotherapy (3D-CRT) of prostate cancer. Radiother Oncol. 2003, 69:215-22. 10.1016/j.radonc.2003.08.003

16. Ryu JK, Winter K, Michalski JM, et al.: Interim report of toxicity from 3D conformal radiation therapy (3DCRT) for prostate cancer on 3DOG/RTOG 9406, level III (79.2 Gy). Int J Radiat Oncol Biol Phys. 2002, 54:1036-46. 10.1016/s0360-3016(02)03006-7

17. Emami B: Tolerance of normal tissue to therapeutic radiation . Radiother Oncol. 2013, 1:35-48.

18. Roach M 3rd, Hanks G, Thames H Jr, Schellhammer P, Shipley WU, Sokol GH, Sandler H: Defining biochemical failure following radiotherapy with or without hormonal therapy in men with clinically localized prostate cancer: recommendations of the RTOG-ASTRO Phoenix Consensus Conference. Int J Radiat Oncol Biol Phys. 2006, 65:965-74. 10.1016/j.ijrobp.2006.04.029

19. Cullen J, Elsamanoudi S, Brassell SA, Chen Y, Colombo M, Srivastava A, McLeod DG: The burden of prostate cancer in Asian nations. J Carcinog. 2012, 11:7. 10.4103/1477-3163.94025

20. Man A, Pickles T, Chi KN: Asian race and impact on outcomes after radical radiotherapy for localized prostate cancer. J Urol. 2003, 170:901-4. 10.1097/01.ju.0000081423.37043.b4

21. Kupelian P, Katcher J, Levin H,et al.: External beam radiotherapy versus radical prostatectomy for clinical stage T1-2 prostate cancer: therapeutic implications of stratification by pretreatment PSA levels and biopsy Gleason scores. Cancer J Sci Am.. 1997, 3:78-87.

22. Nakamura K, Mizowaki T, Imada H, et al.: External-beam radiotherapy for localized or locally advanced prostate cancer in Japan: a multi-institutional outcome analysis. Jpn J Clin Oncol. 2008, 38:200-4. 10.1093/jjco/hyn008

23. Hanks GE, Pajak TF, Porter A, et al.: Phase III trial of long-term adjuvant androgen deprivation after neoadjuvant hormonal cytoreduction and radiotherapy in locally advanced carcinoma of the prostate: the Radiation Therapy Oncology Group Protocol 92-02. J Clin Oncol. 2003, 21:3972-8. 10.1200/JCO.2003.11.023 


\section{Cureus}

24. Lawton CA, DeSilvio M, Roach M 3rd, et al.: An update of the phase III trial comparing whole pelvic to prostate only radiotherapy and neoadjuvant to adjuvant total androgen suppression: updated analysis of RTOG 94-13, with emphasis on unexpected hormone/radiation interactions. Int J Radiat Oncol Biol Phys. 2007, 69:646-55. 10.1016/j.ijrobp.2007.04.003

25. Supit W, Mochtar CA, Santoso RB, Umbas R: Outcomes and predictors of localized or locally-advanced prostate cancer treated by radiotherapy in Indonesia. Prostate Int. 2013, 1:16-22. 10.12954/PI.12012

26. Zumsteg ZS, Spratt DE, Romesser PB, et al.: The natural history and predictors of outcome following biochemical relapse in the dose escalation era for prostate cancer patients undergoing definitive external beam radiotherapy. Eur Urol. 2015, 67:1009-16. 10.1016/j.eururo.2014.09.028 